\title{
Spatial discrimination reversal learning in weanling rats is impaired by striatal administration of an NMDA-receptor antagonist
}

\author{
Deborah J. Watson ${ }^{1,2}$ and Mark E. Stanton \\ University of Delaware, Department of Psychology, Newark, Delaware 19716, USA
}

\begin{abstract}
The striatum plays a major role in both motor control and learning and memory, including executive function and "behavioral flexibility." Lesion, temporary inactivation, and infusion of an $\mathrm{N}$-methyl-D-aspartate (NMDA)-receptor antagonist into the dorsomedial striatum (dmSTR) impair reversal learning in adult rats. Systemic administration of MK801 disrupts reversal learning in developing rats, as reported in an earlier work by Chadman et al., but it is not known whether NMDA-receptor function within the dmSTR plays a role in this effect. In Experiment 1, reversal learning was dose-dependently impaired following bilateral dmSTR administration of MK-801 (either 2.5 or $5.0 \mu \mathrm{g}$ ) only during the reversal phase relative to saline in postnatal day (P) 26 rats. In Experiment 2, separate groups of P26 rats were trained on the same reversal learning task, but were administered bilateral dmSTR infusions during acquisition only (MK-SAL), reversal only (SAL-MK), both phases (MK-MK), or neither phase (SAL-SAL). The MK-801 effect was specific to the reversal training phase. The drug did not alter acquisition of the initial discrimination. Analysis of the pattern of errors indicates that dmSTR MK-801 treatment increased perseveration of the choice response trained in acquisition. NMDA receptors in the dmSTR play a role in reversal learning in the weanling rat.
\end{abstract}

\begin{abstract}
Historically, the primary role of the basal ganglia was thought to be a relay in a "motor loop" involving projection of cortical input by way of the thalamus to motor cortex to guide the initiation and control of movement. An additional loop from association cortex to prefrontal cortex (PFC) through the caudate-putamen (striatum) may play a role in learning and memory (Alexander et al. 1986; Packard and Knowlton 2002; Ragozzino 2007b). Fibers also course through the striatum from the mediodorsal (MD) nucleus of the thalamus en route to the medial prefrontal cortex (mPFC), forming the prefrontothalamic system. Damage to the mPFC, striatum, and MD cause similar behavioral impairments in spatialdelayed alternation (SDA) and spatial reversal tasks in adult rats, impairments characterized by an increase in response perseveration (Divac 1971; Kolb 1977; Vicedomini et al. 1982; Packard and Knowlton 2002). Mild impairments in initial acquisition and impaired reversal have been found (Mitchell et al. 1985; Mitchell and Hall 1988). The reversal impairment is region-specific in the striatum, such that lesions of the medial striatum selectively impairs response (left vs. right turn) discrimination reversals but spares brightness (black vs. white) discrimination reversal (Pisa and Cyr 1990). Within the striatum, damage to the dorsomedial striatum (dmSTR) via lesion, temporary inactivation, and infusion of an $\mathrm{N}$-methyl-D-aspartate (NMDA)-receptor antagonist severely impairs reversal learning in adult rats (Palencia and Ragozzino 2004, 2005; Ragozzino 2007a,b). The reversal impairment is characterized by an increase in learning-related (i.e., regressive) errors in adult animals, which suggests an inability to maintain or reliably execute a newly learned response pattern. It is currently unknown whether this role of NMDA-receptor function within the dmSTR extends to reversal learning in weanling rats.
\end{abstract}

\footnotetext{
'Present address: Section of Neurobiology, Center for Learning and Memory, Institute for Neuroscience, The University of Texas at Austin, Austin, TX 78712-0805, USA. ${ }^{2}$ Corresponding author.

E-mail dwatson@psych.udel.edu; fax (302) 831-3645.
}

Article is online at http://www.learnmem.org/cgi/doi/10.1101//m.1448009.
The effects of striatal lesions on learning and memory across ontogeny have yet to be established longitudinally. When tested as adults, early lesions to the caudate nucleus $(\mathrm{CN})$ in neonatal rats ( P8-P10) did not impair SDA, whereas CN lesions in juveniles $(\sim$ P25-P27) and adults ( P150-P160) severely impaired SDA performance (Vicedomini et al. 1982). These findings are very similar to the data seen after early mPFC lesions on delayed response, SDA, and spatial reversals, when rats lesioned as infants are tested as adults (Kolb and Nonneman 1978; Nonneman and Corwin 1981). Combined mPFC and CN lesions in neonatal rats (P7) severely impaired SDA performance in a T-maze and place acquisition and reversal in a water maze, when rats are tested as adults (Vicedomini et al. 1984). These data suggest that the $\mathrm{CN}$ in developing rats is critical for spatial learning, and when damaged can increase response perseveration during adult reversal performance. The present study is the first to examine the role of NMDA receptors in the dmSTR in reversal learning in developing rats.

The NMDA-receptor subunit representation is not the same across the life span. NR1, NR2A, and NR2C subunits are barely detectable at birth and expression increases during development into adulthood, whereas the NR2B and NR2D subunits are the highest at birth and decline during postnatal development, with NR2D barely detectable in the adult brain (Monyer et al. 1994; Cull-Candy et al. 2001; Haberny et al. 2002). The NR1 subunit is expressed throughout the brain, and the NR2 subunits are expressed in forebrain regions, the hippocampus, thalamus, hypothalamus, brainstem, and cerebellum (Monyer et al. 1994; Cull-Candy et al. 2001; Haberny et al. 2002). Some drugs that act on the NMDA-receptor system preferentially bind to specific receptor subunits. For example, MK-801 preferentially binds to NR1/NR2A and NR1/NR2B receptor subtypes, rather than the NR1/NR2C subtype (Cull-Candy et al. 2001). NR2A receptors demonstrate increased contribution to NMDA-receptor function across the life span, and NR2B receptors have decreased contribution across development. Thus, in the weanling age period both subtypes are expressed throughout the forebrain, whereas in 
adulthood the NR2A subtype is primarily expressed. The preferential subunit binding may play a role in the effects of NMDAreceptor antagonism on reversal learning in weanling rats. It may be possible that MK-801 is more potent in the weanling rat brain than in the adult rat brain-leading to age-related differences in performance characterized by more severe impairments (i.e., enhanced response perseveration) in young rats relative to older rats that typically display deficits in learning the new response pattern in the reversal phase (further considered in the Discussion) (Palencia and Ragozzino 2004). Of particular interest to the present study, NMDA-receptor binding increases across development in the neostriatum such that there is little function at P14, but the receptor binding peaks at P28 and then declines to adultlike levels by P60 (Colwell et al. 1998). This decline in binding density across ontogeny may cause developmental changes in behavior during learning and memory tests following NMDAreceptor antagonism.

Learning of the new discrimination during the reversal phase is more difficult than during the initial acquisition phase (Gonzalez et al. 1966; Pisa and Cyr 1990; Pagani et al. 2005; Chadman et al. 2006). Successful reversal learning requires suppression of the initially acquired response habit from the acquisition phase, while learning a new, competing, response habit during the reversal phase (Pisa and Cyr 1990; Dias et al. 1997). Inability to suppress the initially acquired response habit is characterized by an increase in response perseveration (i.e., perseverative errors), whereas inability to learn the new, competing, response habit is characterized by an increase in regressive errors, the "nonperseverative" errors seen later in the reversal session after the initial perseveration period has ceased (Palencia and Ragozzino 2004). Operationally, a perseverative error occurs when three or more errors are made in a "window" of four successive trials. Perseverative errors are most prevalent at the start of the reversal session. After the perseverative bout has ended and a correct choice has been made, regressive errors (or nonperseverative errors) occur when two or fewer errors are made in a window of four trials. In weanling rats, systemic MK-801 administration dose-dependently increased both perseverative and regressive errors during the reversal learning phase (Chadman et al. 2006). Systemic NMDA-receptor antagonism is likely interfering with both processes in weanling rats because the whole brain is targeted, but in adult rats, specific dmSTR NMDA-receptor antagonism interferes with the learning of the new response habit. It is not known whether dmSTR MK-801 administration will increase perseverative or regressive errors in weanling rats. This question is addressed in the present study.

Discrimination and reversal in a Y-maze can be demonstrated in rats as young as P7 (Kenny and Blass 1977). T-maze reversal does not change between P19 and P30 (Pagani et al. 2005; Chadman et al. 2006). Reversal learning in P21-P30 weanling rats is equivalently impaired by systemic administration of MK-801 (dizocilpine maleate), a noncompetitive NMDA-receptor antagonist (Chadman et al. 2006). The present report is the first to examine the role of NMDA-receptor antagonism in the striatum, and specifically, the dorsomedial subregion in this reversal learning deficit in P26 rats. This age is representative of a range of ages during the weanling period (P21, P26, and P30) in which systemically administered MK-801 impaired reversal learning on this task. The effect of MK-801 within the dmSTR on T-maze discrimination reversal learning was evaluated in two experiments. The dose-response effects of dmSTR MK-801 administration on reversal learning in P26 rats were examined in Experiment 1. Whether the MK-801 effect was specific to reversal relative to acquisition was determined in Experiment 2 by administering MK-801 to separate groups before acquisition (MK-SAL), reversal (SAL-MK), both (MK-MK), or neither (SAL-SAL). This experimen- tal design also determined whether the impairment of reversal performance was due to state-dependent learning effects (further described in Experiment 2, below).

\section{Results}

\section{Experiment 1: Administration of MK-801 into the dmSTR only during reversal}

Experiment 1 was designed to evaluate whether an infusion of MK-801 into the dmSTR only during the reversal phase of training would impair T-maze discrimination reversal learning in weanling rats, and whether the impairment was dose-dependent. Three treatment groups received two sessions of spatial discrimination reversal training, an acquisition session, and a reversal session, performed in the morning and afternoon on a single day. During the reversal session, all groups received bilateral infusions in the dmSTR $(0.5 \mu \mathrm{L} / \mathrm{side})$. Separate groups were administered a high dose of MK-801 (5.0 $\mu \mathrm{g} / \mathrm{side})$, a low dose $(2.5 \mu \mathrm{g} / \mathrm{side})$, or vehicle (sterile saline). If striatal receptors are involved in the impairment of reversal by systemic MK-801 administration seen previously (Chadman et al. 2006), then the present experiment should reveal dose-related impairment of reversal learning following dmSTR administration of MK-801.

\section{Histology-cannula placement}

Rats were included in the analysis if the cannulas were located within or near the dorsomedial region of the striatum (two excluded, 41 included; see included cannula placements in Fig. 1). Group sizes were as follows: saline group $(n=15), 2.5 \mu \mathrm{g} \mathrm{MK}-$ 801-dosed group $(n=13)$, and the $5.0 \mu \mathrm{g}$ MK-801 group $(n=13)$.

\section{Body weight}

ANOVA performed on the deprivation weight data did not reveal differences among subjects in different treatment groups $(F<0.23)$ nor at the start of Session $1(F<0.68)$. Body weights at the start of Session 1 were $53.5 \pm 1.12 \mathrm{~g}, 51.7 \pm 1.32 \mathrm{~g}$, and $52.2 \pm 0.96 \mathrm{~g}$ for the saline, $2.5 \mu \mathrm{g}$ and $5.0 \mu \mathrm{g}$ of MK-treated animals, respectively.

\section{Behavioral effects of striatal administration of MK-80I}

\section{Latency}

Latencies were generally low, and effects of independent variables were numerically small (see Table 1 ). A 3 (treatment) $\times 2$ (phase) $\times$

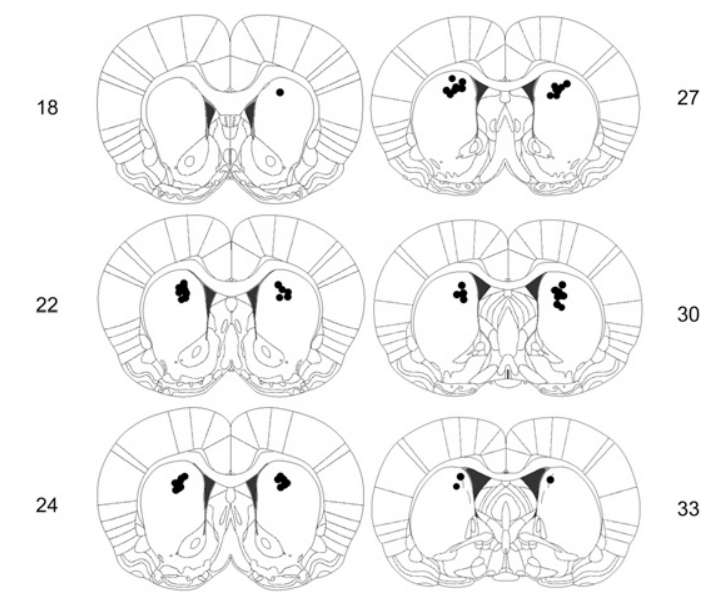

Figure 1. Schematic representation of cannula placements targeted to the dorsomedial region of the striatum in Experiment 1. Numbers represent plates from the stereotaxic atlas of the rat brain (adapted from Paxinos and Watson 2005 and reprinted with permission from Elsevier (02005). 
Table 1. Mean ( \pm SE) of choice run latencies for the three treatment groups on P26 in Experiment 1 as a function of training phase (acquisition or reversal), 12-trial blocks, and dose

\begin{tabular}{|c|c|c|c|c|c|c|c|c|c|}
\hline \multicolumn{5}{|c|}{ ACQUISITION } & \multicolumn{5}{|c|}{ REVERSAL } \\
\hline & Block 1 & Block 2 & Block 3 & Block 4 & Block 1 & Block 2 & Block 3 & Block 4 & $n$ \\
\hline $\begin{array}{l}\mathrm{SAL} \\
\mathrm{MK}-2.5 \\
\mathrm{MK}-5.0\end{array}$ & $\begin{array}{l}6.298 \pm 0.902 \\
5.538 \pm 0.620 \\
5.419 \pm 1.161\end{array}$ & $\begin{array}{l}3.489 \pm 0.238 \\
6.424 \pm 1.301 \\
4.067 \pm 0.469\end{array}$ & $\begin{array}{l}3.225 \pm 0.215 \\
3.884 \pm 0.482 \\
3.168 \pm 0.214\end{array}$ & $\begin{array}{l}3.061 \pm 0.185 \\
3.250 \pm 0.265 \\
2.973 \pm 0.139\end{array}$ & $\begin{array}{l}3.255 \pm 0.222 \\
3.150 \pm 0.157 \\
2.879 \pm 0.111\end{array}$ & $\begin{array}{l}3.495 \pm 0.464 \\
3.102 \pm 0.214 \\
2.820 \pm 0.100\end{array}$ & $\begin{array}{l}2.802 \pm 0.072 \\
2.822 \pm 0.169 \\
2.722 \pm 0.096\end{array}$ & $\begin{array}{l}2.935 \pm 0.146 \\
2.788 \pm 0.162 \\
2.608 \pm 0.037\end{array}$ & $\begin{array}{l}15 \\
13 \\
13\end{array}$ \\
\hline
\end{tabular}

4 (12-trial blocks) repeated-measures ANOVA performed on the latency data revealed significant main effects of phase $\left(F_{(1,38)}=\right.$ $35.93, P<0.0001)$; blocks $\left(F_{(3,114)}=20.07, P<0.0001\right)$, as well as interactions of Phase $\times$ Blocks $\left(F_{(3,114)}=11.35, P<0.0001\right)$ and Phase $\times$ Blocks $\times$ Treatment $\left(F_{(6,114)}=2.60, P<0.021\right)$. In general, reversal latencies were faster than acquisition latencies (Acq: 4.223 $\pm 0.202 \mathrm{sec}$, Rev: $2.957 \pm 0.060 \mathrm{sec}$, respectively) and latency improved across blocks, especially in Blocks 1-2 in acquisition (B1: $5.779 \pm 0.522 \mathrm{sec}, \mathrm{B} 2: 4.603 \pm 0.479 \mathrm{sec}$, respectively) relative to reversal (B1: $3.102 \pm 0.102 \mathrm{sec}, \mathrm{B} 2: 3.156 \pm 0.187 \mathrm{sec}$, respectively). Newman-Keuls post-hoc analyses of the Phase $\times$ Blocks $\times$ Treatment interaction revealed that the 2.5- $\mu \mathrm{g}$ MK-treated animals were significantly slower than the saline-treated animals $(P<$ $0.0001)$ and 5.0- $\mu$ g MK-treated animals $(P<0.0004)$, which did not differ $(P<0.71)$, but only on Block 2 of acquisition. It is difficult to interpret this treatment effect in which the latency effects were limited to a single block in only the low-dose group. Additionally, the latency effects do not match the effects of MK801 on percent correct choice (see below).

\section{Percent correct choice}

The percent correct choice data are shown as a function of drug treatment (saline, $2.5 \mu \mathrm{g}$, and 5.0- $\mu \mathrm{g}$ MK-801-treated animals), and 12 -trial blocks in Figure 2. There were no differences in acquisition performance (as expected, because all groups received saline in acquisition). During reversal, the saline-administered subjects readily acquired the task, as seen by the steady improvement in performance across blocks to acquisition performance levels by the end of reversal training. In contrast, performance of the $2.5-\mu \mathrm{g} \mathrm{MK}-$ 801 group was moderately impaired and the $5.0-\mu \mathrm{g}$ MK-801 group never performed above chance levels throughout reversal training.

A 3 (treatment) $\times 2$ (phase) $\times 4$ (blocks) mixed-factorial ANOVA yielded main effects for treatment $\left(F_{(2,38)}=13.89, P<\right.$ $0.0001)$; phase $\left(F_{(1,38)}=43.39, P<0.0001\right)$; blocks $\left(F_{(3,114)}=196.93\right.$, $P<0.0001)$; Blocks $\times$ Treatment $\left(F_{(6,114)}=3.57, P<0.003\right)$; Phase $\times$ Treatment $\left(F_{(2,38)}=14.69, P<0.0001\right)$. More importantly, a Phase $\times$ Blocks $\times$ Treatment interaction was found $\left(F_{(6,114)}=\right.$ $7.29, P<0.0001)$. Newman-Keuls post-hoc analysis of the Phase $X$ Treatment interaction revealed that the groups did not differ during acquisition, but all three groups differed significantly from each other during reversal (5.0- $\mu \mathrm{g}$ MK-group vs. 2.5- $\mu \mathrm{g}$ MKand saline-treated groups; $P \mathrm{~s}<0.0001$; the latter two groups, $P<0.028$ ). Newman-Keuls post-hoc analysis of the Phase $X$ Blocks $\times$ Treatment interaction revealed that the 5.0- $\mu \mathrm{g}$ MKtreated animals had a lower percent correct choice during Blocks 1-4 of reversal relative to saline-treated animals $(P \mathrm{~s}<0.017)$; and Blocks 2-4 of reversal relative to the $2.5-\mu \mathrm{g}$ MK-treated animals $(P s<0.002)$. Finally, the $2.5-\mu \mathrm{g}$ MK-treated group significantly differed from the SAL group in Blocks $1-2(P<0.003)$. Thus, a clear dose-response effect was found during reversal training.

\section{Error-type analysis}

In order to characterize the types of errors made after MK-801 infusion, independent ANOVAs were performed on the trials to criterion (TTC), total errors, perseverative errors, and regressive errors (Fig. 3). A main effect of treatment was found in the measures of TTC, total errors, and perseverative errors (TTC: $F_{(2,38)}=15.24, P<0.0001$; total errors: $F_{(2,38)}=16.78, P<0.0001$; perseverative errors: $\left.F_{(2,38)}=12.30, P<0.0001\right)$, reflecting an increase in the 5.0- $\mu \mathrm{g}$ MK-treated animals, relative to the $2.5-\mu \mathrm{g}$ MK- and saline-treated animals, which did not differ in perseverative errors $(P<0.17)$ and marginally differed in TTC and total errors $(P s<0.09)$. No effect of treatment was found for regressive errors, $F<0.06$. This suggests that MK-801 treatment increased perseveration at the outset of reversal but did not alter the rate of learning the discrimination at later stages of reversal.

\section{Experiment 2: State-dependent effects of intrastriatal administration}

Reversal learning performance in Experiment 1 was dosedependently impaired following dmSTR administration of MK801. Experiment 2 was designed to determine the specificity of the drug effect on reversal learning, as well as the role of MK-801 blockade on initial learning during acquisition. Poor acquisition performance could suggest a generalized impairment in spatial learning or in sensory, motor, or motivational processes necessary for acquisition of the task. Reversal performance in Experiment 1 could also be related to drug-related cues that may change the internal environment between training phases (i.e., statedependent learning). In young rats, alterations in contextual cues between training phases enhances reversal performance (Moye et al. 1992; Pagani et al. 2005). If the reversal performance is influenced by state-dependent learning effects, we would expect to see the larger impairment in Group MK-MK relative to Group SAL-MK. Systemic MK-801 administration does not statedependently impair reversal performance in weanling rats; therefore, we did not predict that dmSTR MK-801 administration would produce state-dependent learning effects (Chadman et al. 2006).

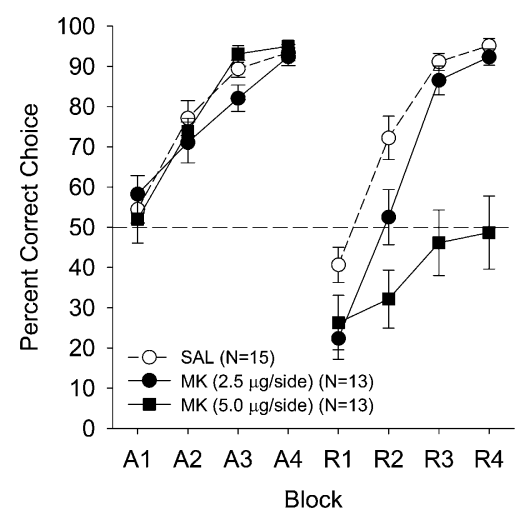

Figure 2. Mean ( $\pm \mathrm{SE}$ ) percentage of correct responses for the three treatment groups on P26 in Experiment 1 as a function of training phase (acquisition or reversal), 12-trial blocks, and dose. During the reversal phase only, the dmSTR treatment groups were vehicle (saline: $O$ ), or one of two drug doses (2.5- $\mu \mathrm{g} \mathrm{MK-801:} \mathrm{\bullet ;} \mathrm{5.0- \mu g} \mathrm{MK-801:} \mathrm{D).} \mathrm{All} \mathrm{treatment}$ groups received saline infusions during acquisition. Dashed line at $50 \%$ indicates chance performance. 

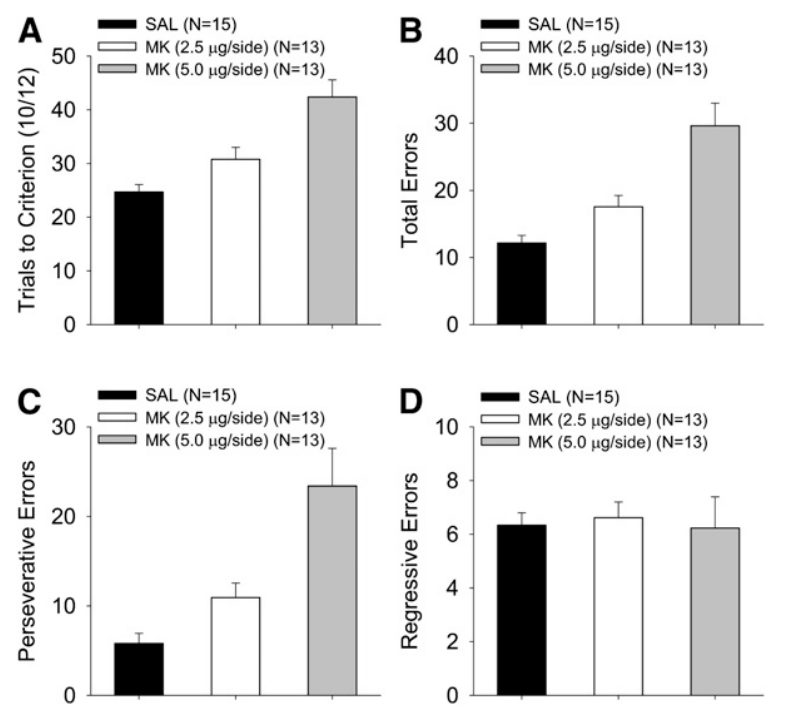

Figure 3. Analysis of trials to criterion (TTC) and error types during reversal for P26 rats as a function of dose of MK-801 in Experiment 1. Mean $( \pm \mathrm{SE})$ for TTC $(A)$, total errors $(B)$, perseverative errors $(C)$, and regressive errors $(D)$.

The lowest effective dose of MK-801 from the previous experiment was used in Experiment 2. This dose was chosen because only this MK group achieved asymptotic levels of reversal performance similar to saline-treated controls. All groups received bilateral infusions into the dmSTR $(0.25 \mu \mathrm{L} / \mathrm{side})$. Groups of rats received bilateral MK-801 (2.5 $\mu \mathrm{g} /$ side) infusions during acquisition only (MK-SAL), reversal only (SAL-MK), both phases (MK$\mathrm{MK})$, or neither phase (SAL-SAL). In order to minimize the possibility that the drug was spreading to brain areas outside $\mathrm{dmSTR}$, a lower volume was selected for the $2.5-\mu \mathrm{g}$ drug dose; however, the lower volume necessitated a higher concentration to be administered (see Materials and Methods). If NMDA-receptor involvement is specific to the reversal learning phase, then acquisition performance will not be impaired, but dmSTR MK801 administration will impair reversal regardless of acquisition drug treatment. Improved reversal performance in the groups that experienced a change in drug condition across the acquisition and reversal phases (i.e., the MK-SAL and SAL-MK groups) would confirm the state-dependent learning hypothesis.

\section{Histology-cannula placement}

Rats were included in the analysis if the cannulas were located within or near the borders of the dorsomedial region of the striatum (four excluded, 55 included; see included cannula placements in Fig. 4). Thus, group sizes were as follows: SAL-SAL group $(n=11)$, SAL-MK group $(n=14)$, MK-SAL group $(n=14)$, and MKMK group $(n=16)$.

\section{Body weight}

A 2 (acquisition treatment) $\times 2$ (reversal treatment) mixedfactorial ANOVA determined that body weights did not differ across groups at deprivation $(F \mathrm{~s}<1.34)$ or at Session $1(F \mathrm{~s}<0.50)$. Body weights at Session 1 were $49.9 \pm 1.19 \mathrm{~g}, 49.6 \pm 1.37 \mathrm{~g}, 50.3 \pm$ $1.03 \mathrm{~g}$, and $50.8 \pm 0.96 \mathrm{~g}$ for the SAL-SAL-, SAL-MK-, MK-SAL-, and MK-MK-treated animals, respectively.

\section{Behavioral effects of striatal administration of MK-801}

\section{Latency}

A 2 (acquisition treatment) $\times 2$ (reversal treatment) $\times 2$ (phase) $\times$ 4 (12-trial blocks) repeated-measures ANOVA performed on the latency data (see Table 2$)$ revealed main effects of blocks $\left(F_{(3,153)}=\right.$ 36.27, $P<0.0001)$, as well as interactions between Phase $\times$ Acquisition treatment $\left(F_{(1,51)}=5.38, P<0.024\right)$, Phase $\times$ Blocks $\left(F_{(3,153)}=13.53, P<0.0001\right)$, and Phase $\times$ Blocks $\times$ Acquisition treatment $\left(F_{(3,153)}=4.65, P<0.004\right)$. Latency was slightly higher during the first block of acquisition (B1: $3.54 \pm 0.124 \mathrm{sec}$ ) relative to reversal (B1: $2.99 \pm 0.101 \mathrm{sec}$ ). Newman-Keuls analyses of the Phase $\times$ Acquisition treatment interaction revealed that the SAL group had slower latencies in acquisition relative to reversal $(P<$ 0.03 ) and phase comparisons for the other groups were not significant (Ps $>0.19$ ). Newman-Keuls analyses of the Phase $\times$ Blocks $\times$ Acquisition treatment interaction revealed that the SAL group had slower latencies in Block 1 of acquisition relative to the MK group, and faster latencies in Block 1 of reversal relative to the MK group ( $P$ s $<0.006)$; no other comparisons between treatment groups were significant. Differences in latency were numerically small and treatment effects on latency did not correspond with treatment effects on percent correct choice.

\section{Percent correct choice}

The percent correct choice data are shown as a function of drug treatment and 12-trial blocks in Figure 5. MK-801 treatment during acquisition did not alter performance during acquisition or reversal. Groups treated with MK-801 during reversal showed impaired performance during the reversal phase relative to those treated with saline during reversal. The groups treated with MK only during reversal (SAL-MK) in Experiment 2 showed a slightly lower percent correct choice ( $\sim 15 \%$ in each block) in comparison to their counterparts in Experiment 1. This minor difference is likely due to the change in volume and concentration between the two studies (see Materials and Methods).

A 2 (acquisition treatment) $\times 2$ (reversal treatment) $\times 2$ (phase) $\times 4$ (12-trial blocks) mixed-factorial ANOVA yielded main effects for reversal treatment $\left(F_{(1,51)}=14.24, P<0.0004\right)$, phase $\left(F_{(1,51)}=58.81, P<0.0001\right)$, and blocks $\left(F_{(3,153)}=155.04, P<\right.$ $0.0001)$. ANOVA also revealed significant interactions of Phase $\times$ Blocks $\left(F_{(3,153)}=7.77, P<0.0001\right)$ and Phase $\times$ Reversal treatment $\left(F_{(1,51)}=13.72, P<0.0005\right)$. The interaction of Phase $\times$ Blocks $\times$ Reversal treatment approached significance $\left(F_{(3,153)}=2.17, P<\right.$ $0.09)$. There was a marginal main effect of acquisition treatment $\left(F_{(1,51)}=3.42, P<0.07\right)$, but there were no significant interaction

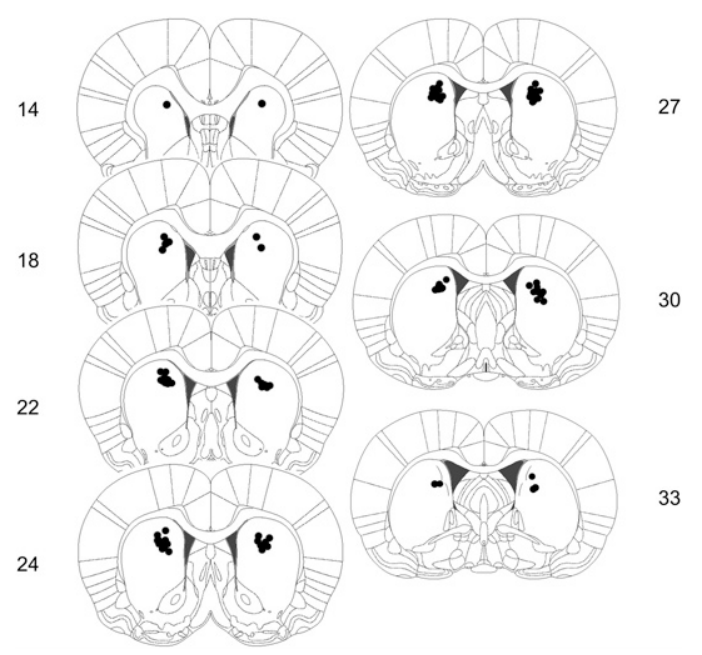

Figure 4. Schematic representation of cannula placements targeted to the dorsomedial region of the striatum in Experiment 2. Numbers represent plates from the stereotaxic atlas of the rat brain (adapted from Paxinos and Watson 2005 and reprinted with permission from Elsevier @2005). 
MK-801, striatum, and reversal learning

Table 2. Mean ( \pm SE) of choice run latencies for the four treatment groups on P26 in Experiment 2 as a function of training phase (acquisition or reversal), 12-trial blocks, and dose

\begin{tabular}{|c|c|c|c|c|c|c|c|c|c|}
\hline \multicolumn{5}{|c|}{ ACQUISITION } & \multicolumn{5}{|c|}{ REVERSAL } \\
\hline & Block 1 & Block 2 & Block 3 & Block 4 & Block 1 & Block 2 & Block 3 & Block 4 & $n$ \\
\hline SAL-SAL & $3.667 \pm 0.167$ & $3.116 \pm 0.179$ & $2.872 \pm 0.114$ & $2.704 \pm 0.043$ & $2.835 \pm 0.111$ & $3.052 \pm 0.273$ & $2.691 \pm 0.071$ & $2.762 \pm 0.112$ & 11 \\
\hline SAL-MK & $3.976 \pm 0.413$ & $3.106 \pm 0.145$ & $2.847 \pm 0.068$ & $2.858 \pm 0.106$ & $2.717 \pm 0.051$ & $2.779 \pm 0.114$ & $2.671 \pm 0.057$ & $2.568 \pm 0.027$ & 14 \\
\hline MK-SAL & $3.331 \pm 0.136$ & $2.965 \pm 0.062$ & $2.827 \pm 0.043$ & $2.692 \pm 0.039$ & $3.342 \pm 0.339$ & $3.405 \pm 0.336$ & $3.244 \pm 0.384$ & $2.926 \pm 0.198$ & 14 \\
\hline MK-MK & $3.269 \pm 0.127$ & $2.931 \pm 0.121$ & $2.703 \pm 0.041$ & $2.736 \pm 0.081$ & $3.033 \pm 0.139$ & $2.791 \pm 0.086$ & $2.577 \pm 0.025$ & $2.662 \pm 0.062$ & 16 \\
\hline
\end{tabular}

effects involving acquisition treatment $(F \mathrm{~s}<1.92)$. Post-hoc analysis of the Phase $\times$ Reversal treatment interaction with Newman-Keuls revealed no effect of reversal treatment on acquisition performance $(P>0.76)$, whereas during reversal MK-801 significantly impaired performance relative to saline treatment $(P<0.0001)$.

In order to further characterize these treatment effects, 2 (acquisition treatment) $\times 2$ (reversal treatment) $\times 4$ (blocks) ANOVAs were performed separately on each training phase. During the acquisition training phase, only a main effect of blocks was found $\left(F_{(3,153)}=55.81, P<0.0001\right)$, indicating that all treatment groups acquired the discrimination at the same rate. During the reversal training phase, ANOVA revealed main effects of reversal treatment $\left(F_{(1,51)}=21.82, P<0.0001\right)$ and blocks $\left(F_{(3,153)}=98.04, P<0.0001\right)$, as well as a marginal main effect of acquisition treatment $\left(F_{(1,51)}=3.97, P<0.052\right)$, and a marginal Blocks $\times$ Reversal Treatment interaction $\left(F_{(3,153)}=2.65, P<0.051\right)$. To further probe whether the marginal acquisition treatment effect on reversal performance was "carried" by the MK-MK treatment group, separate ANOVAs involving each reversal-dose condition did not reveal acquisition treatment effects (MK-SAL vs. SAL-SAL $[P=0.17]$, and SAL-MK vs. MK-MK $[P=0.16])$. Thus, MK801 treatment effects are specific to the reversal learning phase.

Striatal MK-801 administration did not have a statedependent effect. A stimulus change (such as a change in drug administration) between phases is expected to improve reversal performance. The MK-SAL group did not perform better during the reversal phase relative to the SAL-SAL group and Group SALMK did not perform better than Group MK-MK. Thus, statedependency learning effects cannot explain the effect of MK-801 on reversal. Learning during the reversal phase, but not during the initial discrimination, is more sensitive to NMDA-receptor antagonism within the dmSTR.

\section{Error-type analysis}

MK-801 treatment during reversal increased TTC, total errors, perseverative errors, and regressive errors (Fig. 6). Separate ANOVAs revealed a main effect of reversal treatment for TTC $\left(F_{(1,51)}=13.70, P<0.0005\right)$, total errors $\left(F_{(1,51)}=21.72, P<0.0001\right)$, and perseverative errors $\left(F_{(1,51)}=21.79, P<0.0001\right)$, and a marginally significant main effect for regressive errors $\left(F_{(1,51)}=3.15, P<\right.$ $0.082)$. For total and perseverative errors, the main effect of acquisition treatment was marginal (total errors: $F_{(1,51)}=3.94$, $P<0.052$; perseverative errors: $\left.F_{(1,51)}=3.82, P<0.056\right)$. However, these acquisition treatment effects are likely "carried" by the MKMK treatment group, because there was no effect of acquisition dose on total errors or perseverative errors in separate ANOVAs involving each reversal-dose condition (MK-SAL vs. SAL-SAL and SAL-MK vs. MK-MK; all Ps > 0.12). No other statistical effects were significant $(F \mathrm{~s}<2.33)$. MK-801 treatment during the reversal phase enhanced the amount of perseveration on the direction trained in acquisition, which generally impaired reversal performance relative to vehicle treated animals.

\section{Discussion}

Two experiments evaluated the effects of dmSTR NMDA-receptor antagonism on T-maze discrimination reversal learning in developing rats. In Experiment 1, dmSTR administration of MK-801 severely disrupted reversal learning performance in P26 rats in a dose-dependent manner. In Experiment 2, MK-801 (2.5 $\mu \mathrm{g})$ impaired learning only when it was administered during the reversal learning phase. Learning during the acquisition phase was unaffected by MK-801 administration. Impairments in discrimination learning, per se, or in sensory, motor, or motivational processes, or state-dependent learning effects, cannot explain the impairment of reversal learning by MK-801.

Spatial discrimination and its reversal have been studied in developing rats between P7 and P30 (Kenny and Blass 1977; Green and Stanton 1989; Pagani et al. 2005). In our laboratory no developmental change in performance is seen between P19 and P30 (Pagani et al. 2005; Chadman et al. 2006). Systemically administered MK-801 (0.10 mg/kg, i.p.) impairs reversal learning, but not initial acquisition, to a similar extent in P21-P30 rat pups, and the pattern of effects across doses and in the state-dependentlearning design are very similar to the findings reported here with localized dmSTR administration (Chadman et al. 2006). This suggests that NMDA-receptor antagonism within dmSTR is sufficient to account for the effects of systemic drug administration established previously (Chadman et al. 2006). These findings are also the first to show that dmSTR NMDA-dependent plasticity is involved in reversal learning in weanling rats.

A select few studies have examined the effects of lesions or dmSTR infusion of NMDA-receptor antagonists on reversal learning in adult rats (Palencia and Ragozzino 2004; Ragozzino 2007a). A region-specific impairment in reversal learning of a turn discrimination (left vs. right) in a plus-maze was found in adult rats with ibotenic lesions of the medial striatum relative to the lateral

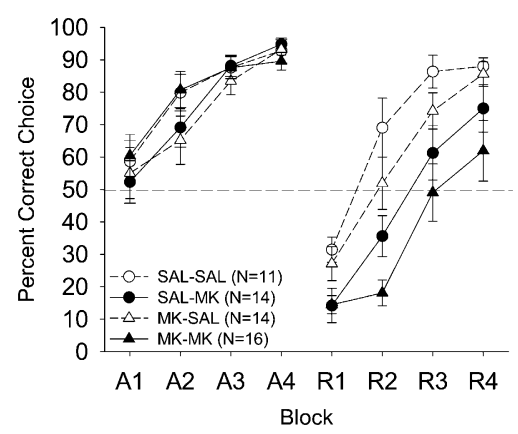

Figure 5. Mean ( $\pm \mathrm{SE})$ percentage of correct responses for the four MK$801(2.5-\mu \mathrm{g})$ treatment groups in Experiment 2 on P26 as a function of training phase (acquisition or reversal), 12-trial blocks, and treatment. The treatment groups were saline-saline (SAL-SAL: O), saline-MK-801 (SALMK: ๑), MK-801-saline (MK-SAL: $\triangle$ ), or MK-801-MK-801 (MK-MK: $\mathbf{\Delta}$ ). Dashed line at $50 \%$ indicates chance performance. 

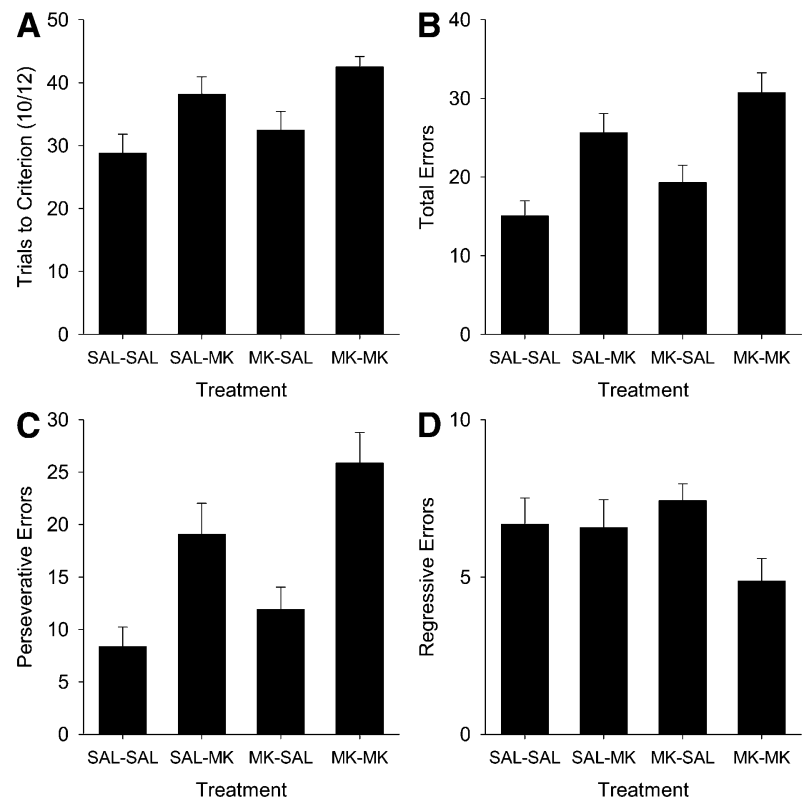

Figure 6. Analysis of TTC and error types during reversal for P26 rats as a function of dose of MK-801 in Experiment 2. Differences were not found between a first (SAL-MK) or second (MK-MK) infusion of MK-801 during reversal. Mean ( \pm SE) for TTC $(A)$, total errors $(B)$, perseverative errors $(C)$, and regressive errors $(D)$. See text for further explanation.

striatum (Pisa and Cyr 1990). Initial studies by Ragozzino established a role for the dmSTR in turn discrimination reversal learning in a modified plus-maze, but not its acquisition, using temporary inactivation with bupivacaine (Ragozzino et al. 2002a). The reversal deficit was not due to response perseveration, but to an inability to learn the new strategy in reversal. Infusion of AP-5, a competitive NMDA-receptor antagonist, into the dmSTR dose-dependently impairs performance selectively in the reversal learning phase in a turn discrimination task, while leaving acquisition performance intact. The deficit was due, again, to impaired maintenance of the newly acquired response habit in reversal, as suggested by an increase in regressive, but not perseverative errors (Palencia and Ragozzino 2004). A subset of subjects at the highest dose of AP-5 in their study displayed an increase in response perseveration. Ragozzino has demonstrated a region-specific double-dissociation between acquisition vs. reversal learning and the dorsolateral vs. dorsomedial striatum, respectively (Palencia and Ragozzino 2005). These findings are in contrast with the present finding that MK-801 increased perseverative but not regressive errors. The discrepancy could reflect differences in the age of subjects tested in the studies (adult vs. weanling rats), or differences in testing apparatus (e.g., T-maze vs. modified plus-maze), experimental methods, or pharmacological agents. The possible role of age differences might be related to the development of the NMDA-receptor system in the striatum of weanling and adult rats. NMDA receptor-binding density measured with autoradiography-labeled MK-801 in the neostriatum increases between P3 and P7 and again between P14 and P21, peaks on P28, and then decreases into adulthood (Colwell et al. 1998). Increased binding density in weanlings may increase the potency of NMDA-receptor antagonists, and this might cause perseverative errors to increase at the expense of regressive errors at this age. As binding density decreases with age, the reversal deficit may shift from the perseverative measure to the regressive error measure in adult rats. Except for this difference in the type of error affected, the impairment of reversal learning following
dmSTR NMDA-receptor antagonism in weanling rats is consistent with the findings in adult rats.

The findings from the present report suggest that NMDAreceptor function within the dmSTR is necessary for reversal learning in P26 rats. Electrolytic lesions of the caudate-putamen in adult rats cause deficits in spatial reversals and enhances response perseveration (Kolb 1977; Mitchell et al. 1985; Mitchell and Hall 1988). Similar results have been found after lesions of the hippocampus, mPFC, and mediodorsal thalamus (Divac 1971; Kolb et al. 1974; Nonneman et al. 1974; Kolb 1977), suggesting that there may be anatomical links between these structures and the striatum that are relevant to spatial reversal learning. The literature regarding the ontogeny of learning and memory functions within the striatum is sparse and is confined to rats tested as adults following neonatal lesions (Vicedomini et al. 1982, 1984). As mentioned previously in text, neonatal lesions (P8-P10) did not impair SDA performance, but juvenile (P25-P27) and adult (P150-P160) lesions severely impaired SDA performance in adulthood (Vicedomini et al. 1982). Although SDA and T-maze reversal are different tasks, this study suggests that learning and memory functions in P25 rats resemble those of adult rats. The present study is the first to directly demonstrate the role of the striatum, specifically NMDA-receptor function, in reversal learning in weanling rats. This finding sets the stage for future investigations concerning when and how this role emerges during postnatal development in the rat.

\section{Materials and Methods}

\section{Experiment 1: Administration of MK-801 into the dmSTR only during reversal}

\section{Subjects}

Forty-eight (23 female, 25 male) Long-Evans rat pups derived from 16 litters served as subjects. Litters were housed in the laboratory vivarium with ad libitum access to food and water on a 12:12 $\mathrm{h}$ light-dark cycle (onset at $0700 \mathrm{~h}$ ). Litters were culled to eight pups (usually four males and four females) on P3 (date of birth is P0). A subset of the pups from the 16 litters were used for Experiment 1; the remaining pups were assigned to other ongoing studies. The pups were weaned on P21 and housed with same-sex littermates until surgery on P23. Weaned pups had uninterrupted access to food and water until the onset of behavioral procedures. Subjects were housed individually in cages following surgery for the duration of the experiment. Pups recovered for $1 \mathrm{~d}$ from surgery before the onset of deprivation (see Procedure below). The average weight at deprivation for subjects was $61.5 \pm 0.7 \mathrm{~g}$ (range: 49.0 $75.0 \mathrm{~g})$. Body weights were not different across treatment groups (see Results). A subset of animals $(n=8)$ were untreated controls from another ongoing study that were weighed on P4 and P9; performance of these animals was no different from the other animals that were not weighed on P4 and P9 $(F s<0.53)$, thus data were pooled across this factor.

Five pups were discarded from analysis for failure to select a maze arm within 3 min from the start of the trial for three or more consecutive trials during the forced run acclimation session $(n=2)$ or the first training session $(n=3)$. Of the remaining 43 pups, two pups were excluded from further analysis following histological analysis of cannula placement. These pups were excluded due to incorrect cannula placements in the ventromedial striatum rather than the dorsomedial striatum. Data from the remaining 41 pups are reported.

\section{Surgery}

Our surgical procedure for weanling rat cannula implantation has been described previously (Watson and Stanton 2009; Watson et al. 2009). Commercially obtained cannulas (Guide: 23 gauge; 
Internal: 30 gauge; Plastics One) were implanted bilaterally under stereotaxic guidance in the brains of weanling rats under ketamine/xylazine anesthesia (52.2-60.9 mg/kg ketamine/7.8-9.1 mg/ $\mathrm{kg}$ xylazine in a $0.7-0.85 \mathrm{~mL} / \mathrm{kg}$ injection vol). Buprenorphine $(0.03 \mathrm{mg} / \mathrm{kg}$ in a vol of $0.05 \mathrm{~mL} / 100 \mathrm{gm})$ was administered subcutaneously to alleviate pain during and following the surgical procedure. The dorsal skull surface was exposed and small holes were drilled in the skull based on stereotaxic coordinates adjusted empirically in pilot studies based on an atlas of the developing rat brain (Sherwood and Timiras 1970). Guide cannulas were bilaterally implanted in the dmSTR $(\mathrm{AP}+7.0 \mathrm{~mm}, \mathrm{ML} \pm 2.3 \mathrm{~mm}$, DV $-3.1 \mathrm{~mm}$ ). All AP and ML coordinates were based on interaural coordinates as measured from the horizontal zero plane, such that the ear bars and incisor bar were set to zero (Sherwood and Timiras 1970). Dummy cannulas were inserted into the guide cannula to prevent obstruction until infusions were made. The guide cannulas were secured to the skull with Loctite, and the cannula assembly was secured to the hooks implanted in the skull with dental acrylic at the end of surgery (Gilbert and Cain 1980; Stanton and Freeman 1994). Following antibiotic ophthalmic ointment application, rats were then returned to their home cages with food and water. Rats were monitored and kept warm until they recovered from anesthesia. Rats received $1 \mathrm{~d}$ of recovery prior to the deprivation procedure that started the T-maze protocol. This amount of recovery time has been found sufficient for weanling/ juvenile rats having undergone stereotaxic surgery (Freeman et al. 2005; Watson and Stanton 2009; Watson et al. 2009).

\section{Drugs}

The experiment involved administration of the noncompetitive NMDA-receptor antagonist, dizocilpine (the compound referred to as MK-801). MK-801 was purchased commercially from Tocris. It was dissolved in sterile saline.

\section{Drug infusion procedure}

Five minutes prior to the start of each position habit training session, the rats were infused (see Watson and Stanton 2009). In Experiment 1, vehicle infusions were made before the acquisition session to all treatment groups, and MK-801 or vehicle was administered before the reversal session. Dummy cannulas were removed and an injection cannula was lowered through each guide cannula extending $1 \mathrm{~mm}$ below the guide cannula. The injection cannula was connected to polyethylene tubing attached to a 10- $\mu \mathrm{L}$ Hamilton syringe mounted on a microinfusion pump. MK-801 was dissolved in sterile saline at a concentration of either 5 or $10 \mu \mathrm{g} / \mu \mathrm{L}$ and delivered at a rate of $0.5 \mu \mathrm{L} /$ minute for $1 \mathrm{~min}$, for a total volume of $0.5 \mu \mathrm{L} /$ side. This volume delivered either 2.5 or $5.0 \mu \mathrm{g}$ of MK-801 per side, for the two concentrations of MK801 , respectively. The same volume of saline was used for the control infusions. One minute after infusion, the injection cannula were removed and replaced with the dummy cannulas. Similar MK-801 doses and volumes are used in the literature for learning studies in adult rats (Zhang et al. 2000, 2001; Bast et al. 2003; Levin et al. 2003; May-Simera and Levin 2003) and weanling rats (Watson and Stanton 2009; Watson et al. 2009).

\section{Apparatus}

The apparatus has been described previously (Freeman and Stanton 1991; Watson and Stanton 2009; Watson et al. 2009). Subjects were trained in four automated Plexiglas T-mazes scaled to the size of weanling rats. Briefly, the T-mazes consisted of three equal length arms: a left and a right choice arm that were perpendicular to the start arm. The start arm was separated from a central choice point and the choice point was separated from the choice arms by pneumatically operated guillotine doors.

Computer-controlled syringe pumps that dispensed a light cream reward ("Half \& Half," Cumberland Dairy) were connected to small metal cups that were located at the ends of both the left and right choice arms. When subjects broke a photoelectric beam in front of the feeding cup, the computer recorded the latency to make the response, lowered the maze doors, and when appropriate, activated the syringe pump (and delivered $0.07 \mathrm{~mL}$ of light cream). Intertrial interval (ITI) boxes of clear Plexiglas were used to house subjects between trials.

\section{Design}

T-maze discrimination reversal training consisted of a total of 96 trials (four 12-trial blocks per session) over two sessions (Freeman and Stanton 1991). The experimental design was a 3 (treatment) $\times$ 2 (phase) $\times 4$ (12-trial blocks) mixed-factorial design. The rewarded goal arm, maze, and sex were counterbalanced across treatment groups. Littermates were assigned to treatment groups such that a maximum of one male and one female per litter contributed to a given treatment group.

\section{Procedure}

All subjects underwent the following procedure of deprivation, maze acclimation, and training. The basic position habit discrimination and reversal procedures have been described previously (Freeman and Stanton 1991; Pagani et al. 2005; Chadman et al. 2006). Briefly, subjects were weaned on P21, received cannula implantation on P23, were deprived on P24, acclimated on P25, and trained on P26.

The deprivation procedure began $\sim 16 \mathrm{~h}$ prior to initial T-maze exposure (usually between 1600 and $1700 \mathrm{~h}$ ). Subjects were deprived of food and water, weighed to the nearest gram, and tail-marked for identification. About $0.1 \mathrm{~mL}$ of the light cream reward was infused directly into each animal's mouth and an additional $1 \mathrm{~mL}$ of cream was placed in the spoon secured to one side of the cage. This procedure minimized taste-related neophobia when subjects were presented with the reward later during acclimation and training.

The acclimation procedure began the next day and pups were trained to consume the light cream from the reward cups at the end of each choice arm during two goal-box training sessions $(\sim 800$ and $1200 \mathrm{~h})$ and then to run in the maze during a forcedrun session that followed $(1600 \mathrm{~h})$. No striatal infusions occurred during maze acclimation.

The T-maze discrimination and reversal sessions began at 0800-0900 $\mathrm{h}$ the following day. Intracranial infusions occurred 5 min before the start of each session as described above. All rats received saline prior to the acquisition session. Different treatment groups received saline or MK-801 prior to the reversal session. Subjects were trained in squads of four animals, with two pups assigned to each maze. For each trial, reward was contingent upon choosing the correct arm (either right or left, counterbalanced across subsets of subjects). At the end of the trial, the subject was returned to its ITI box. The pups were run in rotation such that the ITI for a given pup was the trial time for the other pup in the squad ( $\sim 30 \mathrm{sec}$ ). Sessions lasted $\sim 50-55 \mathrm{~min}$. The reversal session began $5 \mathrm{~h}$ after the start of the acquisition session (usually between 1300 and $1400 \mathrm{~h}$ ). This 5-h interval is sufficient for the behavioral effects of systemic morning drug administration of MK-801 to be eliminated, as well as to maintain adequate motivation levels before the reversal session begins (Chadman et al. 2006). Reversal training procedure was identical to acquisition, except that subjects were rewarded for entrance into the opposite goal arm (i.e., if the rewarded arm in acquisition was the left, in reversal, the rewarded arm was the right). The 48 trials in each training session were run consecutively and were divided into four blocks of 12 trials to analyze changes in performance within training sessions. At the end of the afternoon session, subjects were returned to ad libitum access to food and water.

\section{Histological analysis}

Within 24-48 h after completion of behavioral testing, pups were deeply anesthetized with an intraperitoneal injection of a ketamine/xylazine cocktail following a $0.5-\mu \mathrm{L}$ injection of $2 \%$ pontamine sky-blue dye solution through each guide cannula to show the position of the internal cannula tip. Animals were perfused intracardially with saline followed by formalin, brains were removed and postfixed, and the following day, brains were placed in $30 \%$ sucrose in $10 \%$ buffered formalin. After the brains sank, coronal sections $(40 \mu \mathrm{m})$ were taken using a cryostat (Leica 
CM3050 S), mounted on slides, and then counterstained with Neutral Red (1\%). Slides were examined for cannula tip placement.

\section{Data analysis}

During the training sessions, data were collected for each subject, which included body weight, choice run latencies, and the total percent of correct trials per block. These measures were subjected to analysis of variance (ANOVA) and post-hoc paired comparisons (Newman-Keuls). The between-groups variables used in the analysis were sex (male or female), maze (one to four), treatment (2.5 $\mu \mathrm{g}$ MK-801, 5.0 $\mu \mathrm{g}$ MK-801, or saline), and rewarded goal arm (left or right). The within-group variables were phase (acquisition or reversal) and blocks (four blocks of 12 trials). Preliminary ANOVAs, separately examining each factor, did not reveal effects of sex, maze, or rewarded goal arm; thus, ANOVAs are reported with data combined across these factors.

Percent correct choice data were transformed into a total errors calculation, which was then categorized into either perseverative errors or regressive errors (Dias et al. 1997; Ragozzino et al. 2002b; Chadman et al. 2006; Watson and Stanton 2009). Perseverative errors were defined as incorrect choices three or more times in consecutive blocks of four trials. Fewer than three errors were classified as regressive errors. Perseverative errors provide a measure of the inability of the subject to shift away from a previously reinforced discrimination in acquisition, whereas regressive errors provide a measure of the ability to learn a new discrimination in reversal after initial perseveration declines (Ragozzino et al. 2002b). Trials to criterion (TTC, 10 correct responses in 12 trials) were also calculated; if a subject did not meet these criteria, it was assigned the total number of trials for the reversal session (48) (Chadman et al. 2006).

\section{Experiment 2: State-dependent effects of intrastriatal administration}

The methods, apparatus, etc., in Experiment 2 were the same as those detailed for Experiment 1 except where noted below.

\section{Subjects}

Sixty-one (32 female, 29 male) Long-Evans rat pups derived from 14 litters served as subjects. The average weight at deprivation for subjects was $59.1 \pm 0.60 \mathrm{~g}$ (range: 50.0-72.0 g). Body weights were not different across treatment groups (see Results). Subjects were run in squads of four or eight animals with two or four pups assigned to each maze. Squad size did not affect the outcome of the ANOVAs in different treatment groups; thus, reported statistics include all animals regardless of squad size (see Results).

Two pups were discarded from analysis for failure to select a maze arm within $3 \mathrm{~min}$ from the start of the trial for three or more consecutive trials during the first training session. Of the remaining 59 pups, four additional pups were excluded due to incorrect cannula placements in the ventromedial striatum $(n=3)$ or corpus callosum $(n=1)$ rather than the dorsomedial striatum. Data from the remaining 55 pups are reported.

\section{Design}

The experimental design was a 2 (acquisition treatment) $\times 2$ (reversal treatment) $\times 2$ (phase) $\times 4$ (12-trial blocks $)$ mixedfactorial design. As in Experiment 1, data were combined across sex, maze, and direction of acquisition, because these factors failed to reveal effects in preliminary ANOVAs.

\section{Drug infusion procedure}

The drug infusion procedure was the same as Experiment 1, except that MK-801 was dissolved in sterile saline at a concentration of $10 \mu \mathrm{g} / \mu \mathrm{L}$. MK-801 was delivered at a rate of $0.25 \mu \mathrm{L} /$ minute for $1 \mathrm{~min}$, for a total volume of $0.25 \mu \mathrm{L} /$ side. This volume delivered $2.5 \mu \mathrm{g}$ of MK-801 per side. This volume was used to infuse the $2 \%$ pontamine sky-blue dye solution during histological analysis. All other procedures were the same as detailed in Experiment 1.

\section{Acknowledgments}

This research was supported in part by the University of Delaware, NIH grants 1-R01-AA11945 and 1-PO1-HD35466 to M.E.S. and NRSA fellowship, F31 MH079635 to D.J.W. We thank Andrea Kaiser and Nicole Buzin for technical assistance.

\section{References}

Alexander GE, DeLong MR, Strick PL. 1986. Parallel organization of functionally segregated circuits linking basal ganglia and cortex. Annu Rev Neurosci 9: 357-381.

Bast T, Zhang WN, Feldon J. 2003. Dorsal hippocampus and classical fear conditioning to tone and context in rats: Effects of local NMDAreceptor blockade and stimulation. Hippocampus 13: 657-675.

Chadman KK, Watson DJ, Stanton ME. 2006. NMDA receptor antagonism impairs reversal learning in developing rats. Behav Neurosci 120: 10711083.

Colwell CS, Cepeda C, Crawford C, Levine MS. 1998. Postnatal development of glutamate receptor-mediated responses in the neostriatum. Dev Neurosci 20: 154-163.

Cull-Candy S, Brickley S, Farrant M. 2001. NMDA receptor subunits: Diversity, development and disease. Curr Opin Neurobiol 11: 327-335.

Dias R, Robbins TW, Roberts AC. 1997. Dissociable forms of inhibitory control within prefrontal cortex with an analog of the Wisconsin Card Sort Test: Restriction to novel situations and independence from "online" processing. J Neurosci 17: 9285-9297.

Divac I. 1971. Frontal lobe system and spatial reversal in the rat. Neuropsychologia 9: 175-183.

Freeman JH Jr, Stanton ME. 1991. Fimbria-fornix transections disrupt the ontogeny of delayed alternation but not position discrimination in the rat. Behav Neurosci 105: 386-395.

Freeman JH Jr, Rabinak CA, Campolattaro MM. 2005. Pontine stimulation overcomes developmental limitations in the neural mechanisms of eyeblink conditioning. Learn Mem 12: 255-259.

Gilbert ME, Cain DP. 1980. Electrode implantation in infant rats for kindling and chronic brain recording. Behav Brain Res 1: 553-555.

Gonzalez RC, Berger BD, Bitterman ME. 1966. Improvement in habitreversal as a function of amount of training per reversal and other variables. Am J Psychol 79: 517-530.

Green RJ, Stanton ME. 1989. Differential ontogeny of working memory and reference memory in the rat. Behav Neurosci 103: 98-105.

Haberny KA, Paule MG, Scallet AC, Sistare FD, Lester DS, Hanig JP, Slikker W Jr. 2002. Ontogeny of the $N$-methyl-D-aspartate (NMDA) receptor system and susceptibility to neurotoxicity. Toxicol Sci 68: 9-17.

Kenny JT, Blass EM. 1977. Suckling as incentive to instrumental learning in preweanling rats. Science 196: 898-899.

Kolb B. 1977. Studies on caudate-putamen and dorsomedial thalamic nucleus of rat-implications for mammalian frontal-lobe functions. Physiol Behav 18: 237-244.

Kolb B, Nonneman AJ. 1978. Sparing of function in rats with early prefrontal cortex lesions. Brain Res 151: 135-148.

Kolb B, Nonneman AJ, Singh RK. 1974. Double-dissociation of spatial impairments and perseveration following selective prefrontal lesions in rats. J Comp Physiol Psychol 87: 772-780.

Levin ED, Sledge D, Baruah A, Addy NA. 2003. Ventral hippocampal NMDA blockade and nicotinic effects on memory function. Brain Res Bull 61: 489-495.

May-Simera H, Levin ED. 2003. NMDA systems in the amygdala and piriform cortex and nicotinic effects on memory function. Brain Res Cogn Brain Res 17: 475-483.

Mitchell JA, Hall G. 1988. Caudate-putamen lesions in the rat may impair or potentiate maze learning depending upon availability of stimulus cues and relevance of response cues. Q J Exp Psychol B 40: 243-258.

Mitchell JA, Channell S, Hall G. 1985. Response-reinforcer associations after caudate-putamen lesions in the rat: Spatial discrimination and overshadowing-potentiation effects in instrumental learning. Behav Neurosci 99: 1074-1088.

Monyer H, Burnashev N, Laurie DJ, Sakmann B, Seeburg PH. 1994. Developmental and regional expression in the rat brain and functional properties of four NMDA receptors. Neuron 12: 529-540.

Moye TB, Brasser SM, Palmer L, Zeisset C. 1992. Contextual control of conflicting associations in the developing rat. Dev Psychobiol 25: 151-164.

Nonneman AJ, Corwin JV. 1981. Differential effects of prefrontal cortex ablation in neonatal, juvenile, and young adult rats. J Comp Physiol Psychol 95: 588-602.

Nonneman AJ, Voigt J, Kolb BE. 1974. Comparisons of behavioral effects of hippocampal and prefrontal cortex lesions in the rat. J Comp Physiol Psychol 87: 249-260.

Packard MG, Knowlton BJ. 2002. Learning and memory functions of the basal ganglia. Annu Rev Neurosci 25: 563-593. 
Pagani JH, Brown KL, Stanton ME. 2005. Contextual modulation of spatial discrimination reversal in developing rats. Dev Psychobiol 46: 36-46.

Palencia CA, Ragozzino ME. 2004. The influence of NMDA receptors in the dorsomedial striatum on response reversal learning. Neurobiol Learn Mem 82: 81-89.

Palencia CA, Ragozzino ME. 2005. The contribution of NMDA receptors in the dorsolateral striatum to egocentric response learning. Behav Neurosci 119: 953-960.

Paxinos G, Watson C. 2005. The rat brain in stereotaxic coordinates, 5th ed. Elsevier Academic Press, San Diego, CA

Pisa M, Cyr J. 1990. Regionally selective roles of the rats striatum in modality-specific discrimination-learning and forelimb reaching. Behav Brain Res 37: 281-292.

Ragozzino ME. 2007a. The contribution of the medial prefrontal cortex, orbitofrontal cortex, and dorsomedial striatum to behavioral flexibility. Ann N Y Acad Sci 1121: 355-375.

Ragozzino ME. 2007b. Role of the striatum in learning and memory. In Neurobiology of learning and memory (eds. RP Kesner and JL Martinez). Academic Press, Oxford, UK.

Ragozzino ME, Jih J, Tzavos A. 2002a. Involvement of the dorsomedial striatum in behavioral flexibility: Role of muscarinic cholinergic receptors. Brain Res 953: 205-214.

Ragozzino ME, Ragozzino KE, Mizumori SJ, Kesner RP. 2002b. Role of the dorsomedial striatum in behavioral flexibility for response and visual cue discrimination learning. Behav Neurosci 116: 105-115.

Sherwood NM, Timiras PS. 1970. A stereotaxic atlas of the developing rat brain. University of California Press, Berkeley, CA.
Stanton ME, Freeman JH Jr. 1994. Eyeblink conditioning in the infant rat: An animal model of learning in developmental neurotoxicology. Environ Health Perspect 102: (Suppl. 2) 131-139.

Vicedomini JP, Corwin JV, Nonneman AJ. 1982. Behavioral-effects of lesions to the caudate-nucleus or mediodorsal thalamus in neonatal, juvenile, and adult-rats. Physiological Psychology 10: 246-250.

Vicedomini JP, Isaac WL, Nonneman AJ. 1984. Role of the caudate nucleus in recovery from neonatal mediofrontal cortex lesions in the rat. Dev Psychobiol 17: 51-65.

Watson DJ, Stanton ME. 2009. Intrahippocampal administration of an NMDA receptor antagonist impairs spatial discrimination reversal learning in weanling rats. Neurobiol Learn Mem 92: 89-98.

Watson DJ, Herbert MR, Stanton ME. 2009. NMDA receptor involvement in spatial delayed alternation in developing rats. Behav Neurosci 123: 44-53.

Zhang WN, Bast T, Feldon J. 2000. Microinfusion of the non-competitive $N$-methyl-D-aspartate receptor antagonist MK-801 (dizocilpine) into the dorsal hippocampus of Wistar rats does not affect latent inhibition and prepulse inhibition, but increases startle reaction and locomotor activity. Neuroscience 101: 589-599.

Zhang WN, Bast T, Feldon J. 2001. The ventral hippocampus and fear conditioning in rats: Different anterograde amnesias of fear after infusion of $N$-methyl-D-aspartate or its noncompetitive antagonist MK801 into the ventral hippocampus. Behav Brain Res 126: 159-174.

Received April 6, 2009; accepted in revised form July 14, 2009. 


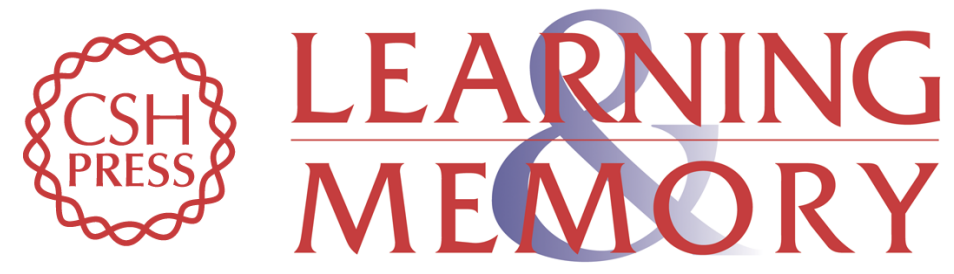

\section{Spatial discrimination reversal learning in weanling rats is impaired by striatal administration of an NMDA-receptor antagonist}

Deborah J. Watson and Mark E. Stanton

Learn. Mem. 2009, 16:

Access the most recent version at doi:10.1101//m.1448009

References This article cites 40 articles, 3 of which can be accessed free at:

http://learnmem.cshlp.org/content/16/9/564.full.html\#ref-list-1

License

Email Alerting Receive free email alerts when new articles cite this article - sign up in the box at the Service top right corner of the article or click here. 